\title{
Clinical Features of Acute Pulmonary Thromboembolism in Younger Patients
}

\author{
Mizuhiro Arima, MD; Tatsuji Kanoh, MD; Atsutoshi Takagi, MD; Kosei Tanimoto, MD; \\ Tetsuya Oigawa, MD; Shigeru Matsuda, MD
}

\begin{abstract}
The incidence of acute pulmonary thromboembolism (APTE) in younger patients is extremely low compared with older patients, so the clinical features of these younger patients with APTE is unknown. In the present study, 8 patients with APTE who were less than 40 years old (YG) were compared with 40 patients who were more than 41 years of age $(\mathrm{OG})$. All YG patients had coagulopathy compared with 3 patients in the $\mathrm{OG}(\mathrm{p}<0.01)$. Deep venous thrombosis (DVT) occurred in all 8 patients in the YG compared with 19 patients in the OG $(\mathrm{p}<0.01)$. A higher incidence of patients whose symptoms occurred gradually was noted in the $\mathrm{YG}(\mathrm{p}<0.05)$. There were no significant differences in clinical characteristics, initial symptoms, past history or other predisposing factors for venous thromboembolism between the 2 groups. Residual pulmonary hypertension was not noted in the YG. However, 1 patient in the YG had recurrent APTE, despite good warfarin control. This study demonstrated the frequency of gradual onset, coagulopathy and clinical signs of DVT in the YG and therefore more careful and long-term observation is necessary in such patients. (Circ J 2003; 67: 330-333)
\end{abstract}

Key Words: Acute pulmonary thromboembolism; Coagulopathy; Deep venous thrombosis; Younger patients

$\mathbf{T}$ he incidence of acute pulmonary thromboembolism (APTE) increases with age, and is low in patients aged less than 40 years compared with the elderly! A Japanese study ${ }^{2}$ showed that the peak age was in the sixth decade and that the incidence of APTE in younger Japanese patients is also very low. The clinical features of younger patients with APTE have not been defined by any study to date, including those of Japanese patients, and there is no known information on the association between the clinical features and age among the patients with APTE. Therefore, the purpose of this study was to describe the clinical characteristics and long-term follow-up of APTE in younger patients.

\section{Methods \\ Patient Population \\ Of 84 patients with APTE who were admitted to Junten- do University Juntendo Urayasu hospital during April 1985 to March 2002, we selected 48 who were willing to under- go further detailed study and long-term follow-up. The study patients were classified into 2 groups according to age of onset: 8 patients with APTE who were less than 40 years old (YG) and 40 patients who were more than 41 years of age (OG). In this study, the clinical diagnosis of APTE was acute onset of symptoms and a mean pulmonary arterial pressure less than $40 \mathrm{mmHg}$. Definitive diagnosis was made from the results of pulmonary perfusion scintigraphy,}

(Received September 11, 2002; revised manuscript received January 10, 2003; accepted January 15, 2003)

Division of Cardiology, Department of Internal Medicine, Juntendo Urayasu Hospital, Juntendo University School of Medicine, Chiba, Japan

Mailing address: Mizuhiro Arima, MD, Division of Cardiology, Department of Internal Medicine, Juntendo Urayasu Hospital, Juntendo University School of Medicine, 2-1-1 Tomioka, Urayasu, Chiba 2790021, Japan computer tomography, magnetic resonance angiography or pulmonary angiography. We investigated clinical symptoms and signs, the presence of underlying diseases or predisposing factors, the presence of deep venous thrombosis (DVT), therapy, prognosis, and clinical course. Patients were classified into 2 types of APTE according to their

Table 1 Clinical Characteristics of Patients With Acute Pulmonary Thromboembolism

\begin{tabular}{lcc}
\hline \hline & $Y G(n=8)$ & $O G(n=40)$ \\
\hline Age (years) & $28.8 \pm 7.0^{* *}$ & $61.7 \pm 11.7$ \\
F/M & $1 / 7^{*}$ & $22 / 18$ \\
Initial symptoms & & \\
$\quad$ Dyspnea & 6 & 31 \\
Chest pain & 4 & 7 \\
Palpitation & 0 & 1 \\
Syncope & 0 & 2 \\
Cardiogenic shock & 1 & 7 \\
Onset & $1 / 7^{*}$ & $23 / 17$ \\
Sudden/gradual & 0 & 6 \\
Recent operation & 0 & 3 \\
Recent catheterization & 0 & 1 \\
Prolonged immobilization & $8^{* *}$ & 3 \\
Coagulopathy & 2 & 0 \\
Protein C deficiency & 1 & 0 \\
Protein S deficiency & $66^{* *}$ & 3 \\
Lupus anticoagulant & 2 & 0 \\
Anticardiolipin antibody & $8^{* *}$ & 19 \\
Deep venous thrombosis & $23.5 \pm 2.1$ & $24.6 \pm 4.8$ \\
Body mass index & 1 & 10 \\
Hypertension & 0 & 6 \\
Cancer & 5 & 11 \\
Smoker & 0 & 3 \\
Heart disease & 0 & 5 \\
Diabetes mellitus & 1 & 2 \\
Hyperlipidemia & & \\
Priat & &
\end{tabular}

Data presented are number of patients. $Y G,<40$ years old; $O G, \geq 41$ years old. ${ }^{*} p<0.05,{ }^{* *} p<0.01$. 
Table 2 Characteristics of the Younger Patients With Acute Pulmonary Thromboembolism

\begin{tabular}{lcccccc}
\hline \hline \multirow{2}{*}{$\begin{array}{l}\text { Patient } \\
\text { no. }\end{array}$} & \multirow{2}{*}{$\begin{array}{c}\text { Age } \\
\text { (years) }\end{array}$} & Sex & Coagulopathy & \multicolumn{2}{c}{ IVC Filter } & \multirow{2}{*}{ Recurrence } \\
\cline { 5 - 6 } 1 & 35 & $M$ & Temporary & Permanent & \\
2 & 20 & $M$ & LAC & + & + & - \\
3 & 32 & $M$ & LAC & - & - & - \\
4 & 40 & $M$ & LAC, $a C L$ & - & - & - \\
5 & 29 & $M$ & $P C$ & - & - & + \\
6 & 25 & $M$ & LAC, $a C L$ & + & - & - \\
7 & 20 & $M$ & LAC & + & - & - \\
8 & 29 & $F$ & LAC & - & - & - \\
\hline
\end{tabular}

PC, protein C deficiency; PS, protein S deficiency; LAC, lupus anticoagulant; aCL, anticardiolipin antibodies; IVC, inferior vena cava.

clinical course: (1) sudden onset of symptoms or (2) gradual onset of symptoms over 3 days.

\section{Examination of Coagulopathy}

The blood concentrations of protein $\mathrm{C}$, protein $\mathrm{S}$, antithrombin III, lupus anticoagulant (LAC), anticardiolipin antibodies (aCL), and homocysteine were measured before thrombolysis or anticoagulation therapy. Protein $\mathrm{C}$ and $\mathrm{S}$ activity, and LAC were measured with activated partial thromboplastin time (APTT) clotting time method. Protein $\mathrm{C}$ and $\mathrm{S}$ antigen concentrations were measured with latex photometric immunoassay and enzyme immunoassay, respectively. aCL were measured with enzyme-linked immunosorbent assay. Antithrombin III and homocysteine were measured with a chromogenic substrate method and highperformance liquid chromatography, respectively.

\section{Evaluation of DVT}

The diagnosis of DVT was confirmed by venography of both legs of all YG patients and of $37 \mathrm{OG}$ patients.

\section{Follow-up Study}

The 2-dimensional and Doppler echocardiograms were examined in all patients upon admission and discharge, and to determine the post discharge pulmonary artery systolic pressure. Other examinations, such as chest roentgenogram, electrocardiogram, pulmonary perfusion scintigraphy and laboratory studies, were performed as required.

\section{Statistical Analysis}

All values were expressed as mean \pm standard deviation. Values in the 2 groups were compared by Fisher's exact test. All significant tests were two-tailed, with $\mathrm{p}<0.05$ considered statistically significant.

\section{Results}

\section{Patient Characteristics and Clinical Features (Table 1)}

The average age of the YG and OG was $28.8 \pm 7.0$ and $61.7 \pm 11.7$ years, respectively, and the female/male ratio was 0.14 and 1.22 , respectively $(\mathrm{p}<0.05)$. Sudden onset of APTE occurred in 1 patient in the YG and in 23 patients in the OG $(\mathrm{p}<0.05)$. All YG patients had coagulopathy compared with 3 patients in the $\mathrm{OG}(\mathrm{p}<0.01)$. In the $\mathrm{YG}, 2$ patients had protein $\mathrm{C}$ deficiency, 6 had LAC, 2 had aCL, and 1 had protein $\mathrm{S}$ deficiency (Table 2). In the OG, only 3 patients had LAC. DVT was observed in all $8 \mathrm{YG}$ patients, but in only 19 patients in the OG $(\mathrm{p}<0.01)$. There were no significant differences in clinical characteristics, initial symptoms, past history or other predisposing factors for
Table 3 Treatment of Acute Pulmonary Thromboembolism

\begin{tabular}{lcc}
\hline \hline Treatment & $Y G(n=8)$ & $O G(n=40)$ \\
\hline Heparin anticoagulation & 8 & 40 \\
Thrombolysis & 6 & 21 \\
Warfarin anticoagulation & 8 & 30 \\
Catheter interventional therapy & 1 & 2 \\
Inferior vena cava filter & & 2 \\
Temporary & $4 * *$ & 4 \\
Permanent & 2 & \\
\hline
\end{tabular}

Data presented are number of patients. $* p<0.05$, **p $<0.01$.

venous thromboembolism between the 2 groups.

\section{Treatment of APTE}

Most patients received specific treatment (Table 3). Intravenous heparin treatment was used in all patients of both groups. In the YG, 6 patients $(75 \%)$ underwent thrombolytic treatment and all patients were treated with warfarin on discharge. In the OG, 21 patients $(53 \%)$ underwent thrombolytic treatment and 30 patients $(75 \%)$ were treated with warfarin on discharge. Catheter interventional therapy was performed in 1 patient in the YG and in 2 in the OG. Emergency surgical thromboembolectomy and percutaneous cardiopulmonary support (PCPS) were not performed in any patient of either group. A temporary inferior vena cave (IVC) filter was inserted in 4 patients in the YG (Table 2), and in 2 patients in the OG $(\mathrm{p}<0.01)$. A permanent IVC filter was implanted in 4 OG patients and in 2 YG patients, one of whom had recurrent APTE and the other had residual massive thrombi in the IVC (Table 2). There were no differences in therapy between the 2 groups except for the use of a temporary IVC filter.

\section{Prognosis of APTE}

Although all YG patients survived, in the OG 3 patients died suddenly in hospital and 3 died after discharge from progression of carcinoma. Residual pulmonary hypertension were noted in 3 patients in the OG, but none in the YG. One patient in the YG had recurrent APTE, despite good warfarin control with a prothrombin time international normalized ratio (INR) of 2.2 (Table 2). In the OG, 3 patients had recurrence: 1 patient with carcinoma who was not given warfarin therapy, one had LAC and another had inadequate warfarin control.

\section{Discussion}

The estimated overall incidence of APTE with or without DVT is 23 per 100,000 in the USA. And the incidence 
increases markedly with age for both males and females from approximately 1 per 10,000 people per year before the age of 40 to 2 in 1,000 per year for those over 70 years of age! The incidence of APTE in Japan is thought to be minimal, and is lower than in the USA ${ }^{3}$ for several possible reasons: racial differences, low body mass index, and no incidence of Factor V Leiden mutation. However, the age distribution of APTE in a Japanese study was similar to Western studies ${ }^{2}$ and also showed that the incidence of APTE is low in Japanese patients younger than 40 years old. In the USA, the incidence of APTE in males is significantly higher than in females, but in another Japanese study, the number of females was higher than that of males. In the present study, the ratio of males to females in the YG was relatively high compared with the $\mathrm{OG}$ and the reason is unclear, but may be caused by age- or sex-related factors involved in the regulation of the coagulation cascade.

Antiphospholipid antibody syndrome is a vascular thrombotic disorder characterized by positive antiphospholipid antibodies, including aCL and LAC, 5 and by an increased risk for arterial and venous thrombosis. The risk for venous thrombosis associated with antithrombin III and deficiencies of proteins $\mathrm{C}$ and $\mathrm{S}$ has been reported, although the protein deficiencies rarely occur. All of the 8 YG patients had coagulopathy compared with 3 patients in the OG. Cancer diagnosed concomitantly or within 1 year of an episode of venous thromboembolism is associated with an advanced stage of cancer and a poor prognosis? and in the 3 OG patients, the cause of death within 1 year after APTE was brain tumor, multiple myeloma, and gallbladder cancer, respectively. There were no cases of cancer in the YG and so there were no other significant predisposing factors for venous thromboembolism in the YG, which suggests that the onset of APTE in young patients may be attributed to coagulopathy.

APTE is generally considered to be associated with DVT and the observed frequency of DVT is approximately $70 \% 8$. In the present OG, the association with DVT was $47.5 \%$, whereas it was $100 \%$ in the YG. In patients aged 30 years or younger who have thrombosis involving the iliac veins, an anomalous IVC is suspected, and those with both an anomaly and thrombosis are at high risk for thrombotic recurrence? However, although abdominal and pelvic computed tomography and venography were routinely performed in all patients of the YG, an anomalous IVC was not found in any of them and so the DVT in the lower extremities was not triggered by this abnormality. The symptoms progressed gradually in most of the patients in the $\mathrm{YG}$, which suggests that as all YG patients had a coagulopathy, a risk factor for venous thrombosis, the APTE can be attributed to the presence of a significant peripheral venous clot burden.

A long-term follow-up study has shown a recurrence of thromboembolism of approximately $3 \%$, despite adequate oral anticoagulation therapy, 10 and a case report presented a patient with no risk factors who experienced recurrence of venous thromboembolism during anticoagulant therapy!1 The present study recorded recurrence of APTE in 1 patient in the YG and in 3 patients in the OG, 2 of whom had inadequate warfarin control and the other was not taking warfarin therapy. Although the YG is very small sample size, the rate of recurrent APTE is high in the YG compared with the OG, despite good warfarin control (INR, 2.2), and is an important finding in terms of therapy and prognosis of younger patients.

Despite the increasing rate of recurrent DVT, IVC filters are effective for the prevention of APTE in patients presumed to be at high risk who have proximal DVT and are on anticoagulant medications $!^{2}$ On the other hand, insertion of IVC filters was not associated with a significant reduction in the 1-year incidence of rehospitalization for APTE 13 A lobulated thrombus on the tricuspid valve of a patient with antiphospholipid antibody syndrome after implantation of IVC filter has been reported, ${ }^{14}$ so the long-term effectiveness of IVC filters is controversial. Therefore, we deliberated whether to implant IVC filters, especially in the YG patients, and only the recurrent case and the patient with massive thrombosis in the IVC were implanted with a permanent IVC filter, concomitant with warfarin therapy. A Japanese study recommends concurrent use of anticoagulation therapy in patients with IVC filters ${ }^{15}$ and we instituted warfarin therapy in all patients with an implanted IVC filters in the absence of contraindications.

One study in the USA demonstrated that the prevalence of chronic pulmonary hypertension was $0.1-0.2 \%$ of patients surviving APTE ${ }^{16}$ and in the present study, residual pulmonary hypertension was diagnosed in 3 OG patients only, which makes the prevalence rate in our study patients higher than that previously reported in the United States. Because there is not a specific Japanese study of pulmonary hypertension caused by APTE, we do not know why the prevalence of chronic pulmonary hypertension was higher in our patient population. In the United States, most cases of chronic pulmonary thromboembolism (CPTE) seem to be related to APTE, but it is unlikely in Japan that patients with APTE would develop CPTE with pulmonary hypertension? There are 2 concepts of CPTE: (1) unsuccessful lysis because of delayed thrombolytic therapy and (2) organized thrombus. One Japanese study demonstrated that coagulopathy, such as protein C deficiency, AT III deficiency and LAC, was more frequent in patients with CPTE than $\mathrm{APTE}^{4}$ In the 3 patients with residual pulmonary hypertension in the OG, lysis would have been unsuccessful because thrombolytic therapy was delayed. On the other hand, although all the YG patients had both coagulopathy and DVT, none had residual pulmonary hypertension, which demonstrates that early diagnosis and adequate therapy are important. One study revealed that the main cause of CPTE was DVT that repeatedly detached and caused chronic thrombosis in the pulmonary artery! ${ }^{16}$ In the present study, all YG patients had DVT and their symptoms progressed gradually. Therefore, accurate diagnosis is demanded and careful follow-up is necessary to determine if younger patients with APTE will develop CPTE.

\section{Study Limitations}

The patients in the present study were selected from one hospital only and the sample size was small. The patients did not have any predisposing factors such as plasma hyperhomocysteinemia, antithrombin III deficiencies or anomalous IVC. There was only 1 female patient in the YG and because deficiencies of proteins $\mathrm{C}$ and $\mathrm{S}$ and antiphospholipid antibody syndrome are not predominant in males, our results can not be generalized.

In the YG, 2 patients (cases 1 and 5) are computer programmers who sit on a chair for long periods, as in economy class syndrome. Another patient (case 6) is a beautician whose job requires standing for a long time, and 2 of the patients (cases 3 and 7) are obese. Those factors may have contributed to the onset of APTE.

This is a retrospective analysis and therefore, detailed 
and long-term follow-up are necessary.

\section{Conclusion}

Our study demonstrated that younger patients with APTE have coagulopathy and the clinical signs of DVT, which occurs gradually. Residual pulmonary hypertension was not a feature of the YG, but despite good warfarin control, one patient in the YG had recurrent APTE. Therefore, careful and long term observation is necessary to determine whether younger patients with APTE will develop CPTE.

\section{References}

1. Anderson FA, Wheeler HB, Goldberg RJ, Hosmer DW, Patwardhan NA, Jovanovic B, et al. A population-based perspective of the hospital incidence and case-fatality rates of deep vein thrombosis and pulmonary embolism. The Worcester DVT study. Arch Intern Med 1991; 151: $933-938$.

2. Kumasaka N, Sakuma M, Shirato K. Clinical features and predictors of in-hospital mortality in patients with acute and chronic pulmonary thromboembolism. Intern Med 2000; 39: 1038-1043.

3. Kumasaka N, Sakuma M, Shirato K. Incidence of pulmonary thromboembolism in Japan. Jpn Circ J 1999; 63: 439-441.

4. Nakamura M, Okada O, Sakuma M, Nakanishi N, Miyahara Y, Yamada N, et al. Incidence and clinical characteristics of chronic pulmonary thromboembolism in Japan compared with acute pulmonary thromboembolism: Results of a multicenter registry in the Japanese society of pulmonary embolism research. Circ J 2002; 66: $257-260$.

5. Hughes GRV. The antiphospholipid syndrome: Ten years on. Lancet 1993; 342: 341-344.

6. Harris EN, Charavi AE, Boey ML. Anticardiolipin antibodies: Detection by radioimmunoassay and association with thrombosis in sys- temic lupus erythematosus. Lancet 1983; ii: 1211-1214.

7. Sorensen HT, Mellemkjaer L, Olsen JH, Baron JA. Prognosis of cancers associated with venous thromboembolism. N Engl J Med 2000; 343: $1846-1850$.

8. Simonneau G, Sors H, Charbonnier B, Page Y, Laaban J-P, Azarian $\mathrm{R}$, et al. A comparison of low-molecular-weight heparin with unfractionated heparin for acute pulmonary embolism. N Engl J Med 1997; 337: $663-669$.

9. Obernosterer A, Aschauer M, Schnedl W, Lipp RW. Anomalies of the inferior vena cava in patients with iliac venous thrombosis. Ann Intern Med 2002; 136: 37-41.

10. Schulman S, Granqvist S, Holmstrom M, Carlsson A, Lindmarker $\mathrm{P}$, Nicol P, et al. The duration of oral anticoagulant therapy after a second episode of venous thromboembolism. N Engl J Med 1997; 336: $393-398$

11. Tanimoto K, Mizushige K, Yukiiri K, Ueda T, Yoshihiro W, Ohmori $\mathrm{K}$, et al. Recurrence of idiopathic thromboembolism during anticoagulant therapy. Jpn Circ J 2001; 65: 755-756.

12. Decousus H, Leizorovicz A, Parent F, Page Y, Tardy B, Girard P, et al. A clinical trial of vena caval filters in the prevention of pulmonary embolism in patients with proximal deep-vein thrombosis. $N$ Engl $J$ Med 1998; 338: 409-415.

13. White RH, Zhou H, Kim J, Romano PS. A population-based study of the effectiveness of inferior vena cava filter use among patients with venous thromboembolism. Arch Intern Med 2000; 160: 2033-2041.

14. Ebato M, Kitai H, Kumakura H, Nakamura Y, Shimizu N, Takeyama Y. Thrombus on the tricuspid valve in a patient with primary antiphospholipid syndrome after implantation of an inferior vena cave filter. Circ J 2002; 66: 425-427.

15. Yazu T, Fujioka H, Nakamura M, Hiraoka N, Yamada N, Ohta M, et al. Long-term results of inferior vena cava filters: Experiences in a Japanese population. Intern Med 2000; 39: 707-714.

16. Jamieson SW, Auger WR, Fedullo PF, Channick RN, Kriett JM, Tarazi RY, et al. Experience and results with 150 pulmonary thromboendarterectomy operations over a 29 -month period. J Thorac Cardiovasc Surg 1993; 106: 116-127. 